\title{
¿El voto directo como derecho humano?: el caso de las elecciones presidenciales de los Estados Unidos de América
}

Sebastián Herrera Castro*

https://doi.org/10.35242/RDE $2021 \quad 32 \quad 13$

Nota del Consejo Editorial

Recepción: 4 de abril de 2021.

Revisión, corrección y aprobación: 5 de julio de 2021.

Resumen: Analiza si el voto directo puede considerarse un derecho humano. Para ello, revisa los instrumentos internacionales con el auxilio de criterios de organismos internacionales pertinentes y doctrina especializada. Reflexiona que los instrumentos universales establecen el requerimiento del voto en igualdad, lo cual no puede cumplirse a cabalidad con elecciones indirectas. En ese sentido, concluye que el voto directo en el sufragio para elegir la jefatura de Gobierno sí es un derecho humano y que puede ser satisfecho aún en los regímenes parlamentarios si esa jefatura del Gobierno es elegida como integrante del parlamento y designada por el mismo cuerpo representativo, ante el cual también es responsable. Estudia específicamente el sistema de las elecciones presidenciales de los Estados Unidos de América, de donde se concluye que el Colegio Electoral impide que haya voto en igualdad y voto directo, por lo que se requiere ajustar el sistema a los estándares contemporáneos de derechos humanos, con miras a asegurar que el voto popular sea el que determine la jefatura del Gobierno, pues se trata de un sistema político en el que la presidencia ostenta fuertes potestades y una alta cuota de poder político.

Palabras clave: Voto directo / Voto indirecto / Elección directa / Elección indirecta / Sistemas electorales / Sistema político / Derecho al sufragio / Derechos políticos / Derechos humanos / Elecciones presidenciales / Estados Unidos.

Abstract: It analyzes whether direct voting can be considered a human right. To do this, it presents a review of international tools with the aid of criteria of the corresponding international bodies and specialized doctrine. It states that the universal instruments established the requirement of equal voting, which could not be fully met with indirect elections. In this regard, it concludes that direct voting in the suffrage to elect the head of government is a human right and that it can be satisfied even in parliamentary regimes if that head of government is elected as a member of parliament and appointed by the same representative body, to which it is also responsible. It specifically studies the system of presidential elections in the United States of America, from which it is concluded that the Electoral College prevents equal voting and direct voting, and it is therefore necessary to adjust the system to contemporary human rights standards, in order to ensure that the popular vote is the one that determines the head of government, since it is a political system in which the presidency has strong powers and a high share of political power.

Key Words: Direct voting / Indirect voting / Direct election / Indirect election / Electoral systems/ Political system / Right to suffrage / Political rights / Human rights / Presidential elections / United States.

\footnotetext{
* Costarricense, bachiller en Derecho por la Universidad de Costa Rica (UCR) y egresado de la Licenciatura en Derecho con mención en Formación de Jueces, correo sherreracastro94@outlook.com. Actualmente labora como técnico judicial encargado de Cooperación Judicial Internacional en la Sala Segunda de la Corte Suprema de Justicia. Estudia Administración Pública y Filología Española. Fue miembro del Tribunal Electoral Estudiantil Universitario (TEEU) de la UCR en 2019, donde también ha fungido como comisionado de honor y editor de la publicación 10 minutos en el período 2020-2021. Presidió el Tribunal Electoral Estudiantil de Derecho (TEED) de la Asociación de Estudiantes de Derecho de la UCR (AED) en el período 2018-2019. Ha participado en la redacción de diversa normativa nacional y universitaria. Sus principales áreas de interés académico y profesional son: derecho constitucional, electoral, administrativo, laboral e internacional.
} 


\section{DERECHOELECTORAL}

\section{INTRODUCCIÓN}

De las seis elecciones presidenciales realizadas en los Estados Unidos de América durante el siglo XXI, dos han resultado en la designación de un presidente que no obtuvo la mayoría del voto popular (National Archives, 2020). Esta situación ha reavivado la discusión sobre la perfectibilidad de ese sistema electoral, especialmente la cuestión del voto indirecto y el Colegio Electoral.

Los Estados Unidos de América son conocidos como la democracia más antigua del mundo, principalmente porque su Constitución - vigente desde 1789- estipula que el pueblo elige de manera periódica a sus gobernantes, específicamente, a quienes ejercen el poder legislativo (consistente en el Congreso, órgano bicameral compuesto por el Senado y la Cámara de Representantes) y el poder ejecutivo (encabezado por la presidencia y la vicepresidencia). Desde entonces, todos los presidentes han sido elegidos mediante sufragio indirecto.

En este sistema, los votos emitidos en primer grado por la ciudadanía se convierten en electores, quienes representan a cada circunscripción electoral -50 estados además del Distrito de Columbia- en el Colegio Electoral, cuerpo encargado de elegir, en segundo grado, a quienes ocuparán la presidencia y la vicepresidencia de la nación.

Si bien este modelo ha servido de inspiración para decenas de democracias en todo el mundo, especialmente en Latinoamérica, el hecho es que la jefatura de estado y de Gobierno no son elegidas de manera directa. En este artículo se aborda esa situación desde una perspectiva jurídico-política de derechos humanos.

En primer término, se busca determinar si es posible considerar el voto directo como un derecho humano, para lo cual se realizará una conceptualización del voto directo y el voto indirecto, luego de lo cual se estudiarán los instrumentos internacionales en materia de derechos políticos, a la luz de la doctrina más relevante. De seguido, se pretende analizar el rasgo de voto indirecto del sistema de elecciones presidenciales de los Estados Unidos de América, para identificar los posibles conflictos con los paradigmas de derechos humanos contemporáneos, tomando en cuenta la legitimidad o aceptación que tiene, actualmente, ese sistema en la población. 


\section{DERECHO ELECTORAL}

\section{CONCEPTUALIZACIÓN DEL VOTO DIRECTO Y EL VOTO INDIRECTO}

El sufragio, como mecanismo por el cual el pueblo elige a sus representantes y gobernantes -en el marco de una democraciaengloba, en términos simples, dos derechos para cada persona ciudadana: la oportunidad de elegir (sufragio activo) y la de ser electa (sufragio pasivo). El voto corresponde a la vertiente activa del sufragio (Brenes y Matarrita, 2012), lo cual debe tenerse presente a la hora de caracterizarlo y juzgar la idoneidad de sus diferentes modalidades.

Una de las primeras discusiones jurídicas sobre el carácter directo o indirecto del voto para elegir a las autoridades políticas se remonta a la Revolución francesa, proceso en el cual se emitieron normas para regular ambos tipos de elección (IIDH,2017). No sobra recordar que estas ideas son centrales en las nociones básicas que informan las discusiones que se tienen, incluso hoy, sobre el tema.

Una concepción contemporánea del voto directo es la ofrecida por FixFierro, citado por Rosales García, quien define: "Voto directo: quiere decir que los candidatos reciben los votos de los ciudadanos sin intermediación de ningún órgano o cuerpo de electores" (2014, p. 111). Esta conceptualización no es solo clara, sino que aporta un criterio diferenciador ente los sistemas de voto directo e indirecto: la existencia o no de un órgano que intermedie la elección entre los votos de la ciudadanía y el resultado final.

El factor del voto directo tiene estrecha relación con la calidad de la democracia, puesto que "mientras mayor sea el número y la jerarquía de los funcionarios sometidos a elección popular, mayor será la oportunidad y efectividad de la participación política democrática, así como el grado de control directo de la población sobre quienes la gobiernan" (IIDH, 2017, p. 123).

Sin embargo, también debe considerarse la intensidad o la cuota de poder político de cada uno de esos cargos de elección popular, ya que tienen distintas implicaciones, en principio, las elecciones de representantes en el poder legislativo que a las de cargos del ejecutivo o el judicial. Pero ello también se verá afectado por la constitución del sistema político: podría considerarse más relevante, por ejemplo, la votación directa para elegir a la jefatura del Gobierno si esta se encuentra en un régimen presidencialista, cuya característica central es la de un poder ejecutivo con fuertes potestades, estabilidad e independencia frente a otros poderes del 


\section{DERECHO EIECTORAL}

Estado; caso contrario al que se da en el marco de un sistema parlamentario, en el que la función ejecutiva se desarrolla de la mano del poder legislativo, sus titulares derivan de este último cuerpo, son responsables ante él e incluso pueden ser removidos por su voluntad.

\section{3. ¿ES EL VOTO DIRECTO UN DERECHO HUMANO?}

Con independencia de lo que señala la doctrina que luego se revisará, las primeras fuentes a estudiar para determinar si puede considerarse derecho humano el carácter directo del voto son los textos de los instrumentos internacionales. En el ámbito universal, las disposiciones vigentes de mayor antigüedad se recogen en la Declaración Universal de Derechos Humanos (Organización de las Naciones Unidas, 1948), cuyo artículo 21 indica:

\section{Artículo 21}

1. Toda persona tiene derecho a participar en el gobierno de su país, directamente $o$ por medio de representantes libremente escogidos.

2. Toda persona tiene el derecho de acceso, en condiciones de igualdad, a las funciones públicas de su país.

3. La voluntad del pueblo es la base de la autoridad del poder público; esta voluntad se expresará mediante elecciones auténticas que habrán de celebrarse periódicamente, por sufragio universal e igual y por voto secreto $u$ otro procedimiento equivalente que garantice la libertad del voto.

Del inciso 3 de ese artículo se extraen algunos atributos básicos del sufragio: periódico, universal, igualitario, secreto y libre. Pero la función de esas cualidades parece ser, en este texto, la de dotar de legitimidad al poder político, pues el sufragio sirve para concretar la voluntad del pueblo en la designación de personas electas para gobernar.

Dos décadas más tarde, el Pacto Internacional de Derechos Civiles y Políticos (Organización de las Naciones Unidas, 1966) reformula esas garantías, concibiéndolas más en función del individuo que de la soberanía nacional: 


\section{DERECHOELECTORAL}

\section{Artículo 25}

Todos los ciudadanos gozarán, sin ninguna de la (sic) distinciones mencionadas en el artículo $2^{1}$, y sin restricciones indebidas, de los siguientes derechos y oportunidades:

a) Participar en la dirección de los asuntos públicos, directamente $o$ por medio de representantes libremente elegidos;

b) Votar y ser elegidos en elecciones periódicas, auténticas, realizadas por sufragio universal e igual y por voto secreto que garantice la libre expresión de la voluntad de los electores;

c) Tener acceso, en condiciones generales de igualdad, a las funciones públicas de su país [énfasis es suplido].

Al comparar ambas normas, puede notarse que la de 1948 concibe el sufragio como un mecanismo para legitimar el poder político partiendo de la voluntad popular, definición más cercana a la soberanía nacional; mientras que la de 1966 entiende las elecciones como "derechos y oportunidades" de cada persona ciudadana. El Comité de Derechos Humanos de la Organización de las Naciones Unidas ha explicado que si bien el artículo 25 del Pacto de Nueva York aquí analizado guarda relación con el derecho de los pueblos a la libre determinación -lo cual se entiende en sentido colectivo-, en realidad protege los derechos individuales de participación política de cada persona ciudadana, por lo que se trata de derechos individuales, plenamente exigibles por consistir en obligaciones internacionales de los Estados (1996, párrs. 2-3).

Según Fox (1992), los derechos de participación política tuvieron, en los inicios del derecho internacional moderno, poca cabida, puesto que amenazaban la noción tradicional de soberanía, que no necesariamente tenía como base la voluntad popular.

Sin embargo, Picado Sotela (2007) sostiene que el consenso doctrinario es el de reconocer a los derechos políticos como derechos humanos, lo cual

\footnotetext{
${ }^{1}$ El artículo 2 expresa: "1. Cada uno de los Estados Partes en el presente Pacto se compromete a respetar y a garantizar a todos los individuos que se encuentren en su territorio y estén sujetos a su jurisdicción los derechos reconocidos en el presente Pacto, sin distinción alguna de raza, color, sexo, idioma, religión, opinión política o de otra índole, origen nacional o social, posición económica, nacimiento o cualquier otra condición social $(\ldots)^{\prime \prime}$.
} 


\section{DERECHO ELECTORAL}

no impide que constituyan una categoría especial con características, principios e instituciones propias.

Establecido el carácter individual de los derechos relativos al voto tutelados en estos instrumentos, debe analizarse si las características dichas se encuentran necesariamente vinculadas con un atributo de voto directo.

El inciso b) del artículo 25 citado establece como requisito el voto en igualdad. Sobre el alcance del atributo de igualdad de las elecciones, el Comité de Derechos Humanos de la ONU comentó:

21. Aunque el Pacto no impone ningún sistema electoral concreto, el sistema electoral vigente en un Estado Parte debe ser compatible con los derechos amparados por el artículo 25 y garantizar y dar efecto a la libre expresión de la voluntad de los electores. Debe aplicarse el principio de un voto por persona $y$, en el marco del sistema electoral de cada uno de los Estados, el voto de un elector debe tener igual valor que el de otro. La delimitación de los distritos electorales y el método de asignación de votos no deben desvirtuar la distribución de los votantes ni comportar discriminación alguna contra ningún grupo, ni tampoco excluir o restringir en forma irrazonable el derecho de los ciudadanos a elegir libremente a sus representantes [resaltado no es del original]. (1996, párr. 21).

En otras palabras, el Pacto no pretende definir las reglas específicas de los sistemas electorales de cada país, pero sí instaura como obligación el hecho de que exista igualdad en el valor del voto de cada persona.

En la publicación Human Rights and Elections, (United Nations Centre for Human Rights, 1994, p. 11), también se resume el elemento de igualdad en la frase "una persona, un voto", y se amplía en cuanto a que las circunscripciones electorales no deben diluir ni descontar los votos de individuos particulares, sino que cada voto debe tener el mismo peso en la elección. En ese mismo texto se hace referencia al Borrador de los Principios generales sobre la libertad y la no-discriminación en asuntos de derechos políticos, documento propuesto en 1962 por la Subcomisión Sobre Prevención de Discriminación y Protección de Minorías, como una iniciativa tendiente a consolidar internacionalmente algunos criterios fundamentales en cuanto a derechos políticos en el contexto democrático. Allí se planteaba una definición del principio de igualdad del sufragio en los siguientes términos: 


\section{DERECHOELECTORAL}

\section{Igualdad del sufragio}

(a) Cada ciudadano tiene derecho a votar en toda elección nacional y demás consultas públicas pertinentes, en iguales términos, y cada voto tendrá el mismo peso.

(b) Cuando el voto se realice con base en distritos electorales, dichos distritos serán establecidos de una manera equitativa, de tal manera que los resultados reflejen de manera exacta y completa la voluntad de las personas electoras.

(c) Para toda elección y consulta pública realizada mediante voto directo, habrá un padrón electoral general, y cada ciudadano será incluido en dicho padrón ${ }^{2}$. (United Nations Centre for Human Rights, 1994, p. 21).

De lo cual se resalta el requerimiento de que todos los votos tengan el mismo peso en la elección, y la necesidad de que, en caso de contar con circunscripciones electorales, aún se deba asegurar que el resultado final refleje la voluntad de la totalidad de las personas electoras.

El anterior criterio se recoge en doctrina contemporánea. Fix-Fierro, citado por Rosales García reflexiona en el siguiente sentido:

La igualdad en el voto tiene un doble significado: por un lado, que los individuos tienen igual número de votos; por el otro, que el valor y el peso de cada voto es aproximadamente el mismo, esto es, cuando existe una proporción similar entre el número de votantes y el número de representantes electos [énfasis es suplido]. (2014, p. 111).

Por su parte, Aragón recuerda que no es suficiente que el sufragio sea universal para que haya una verdadera democracia, sino que: "ha de ser

\footnotetext{
${ }^{2}$ Traducción es provista. El texto original en inglés indica: " $V$. Equiality of suffrage / (a) Every national is entitled to vote in any national election, or other public consultation for which he is eligible, on equal terms, and each vote shall have the same weight. (b) When voting is conducted on the basis of electoral districts, the said districts shall be established on an equitable basis such as would make the results more accurately and completely reflect the will of all the voters. (c) For any election or public consultation held by direct vote there shall be one general election roll, and every eligible national shall be included in that roll".
} 


\section{DERECHO EIECTORAL}

también libre, igual, directo y secreto. El voto de los ciudadanos ha de valer igual, ha de emitirse sin intermediarios [...]" (2007, p. 163).

El tema también figura en publicaciones de organismos especializados. El Instituto Internacional para la Democracia y la Asistencia Electoral (IDEA) señala que debe asegurarse una efectiva representación, lo cual implica que el marco normativo que establece las circunscripciones electorales procure otorgar igual peso para todos los votos ciudadanos (2002). Esto se expresa a través del desarrollo de dos estándares de los sistemas electorales. En primer lugar:

\section{Sufragio universal e igual}

Un claro estándar internacional que debe mantenerse es la garantía de sufragio universal e igual para cada persona ciudadana adulta. El derecho a ser elegida como miembro del poder legislativo u otro cuerpo provincial o local, así como el derecho de ser elegida en la presidencia, $[\ldots]$ debe ser garantizado a todas las personas ciudadanas $[\ldots]^{3}$. (IDEA, 2002, p. 34).

Así como la igualdad de la fuerza de cada voto, lo que se formula con el siguiente criterio:

\section{Igualdad de la fuerza de cada voto}

Los límites deben dibujarse de tal forma que los distritos sean relativamente iguales en cuanto a la fuerza de cada votante, de manera que cada votante pueda emitir un voto de igual peso, en la mayor medida posible. Con circunscripciones electorales de igual cantidad de personas, las personas votantes tendrían un voto con igual peso en la elección de representantes; sin embargo, este ideal no es posible en la práctica, y concurren otros principios que también deben aplicarse ${ }^{4}$. (IDEA, 2002, p. 29).

\footnotetext{
3 Traducción es provista. El texto original en inglés indica: "Universal and equal suffrage / One clear international standard which must be provided for is the guarantee of universal and equal suffrage to each adult citizen. The right to be elected as a member of the legislature or other provincial or local body, as well as the right to be elected president, [...] must be guaranteed to all citizens [...]".

${ }^{4}$ Traducción es provista. El texto original en inglés indica: "Equality of voting strength / Boundaries should be drawn so that constituencies are relatively equal in voter strength, resulting in each voter casting a vote of equal weight to the greatest degree possible. Equi-populous electoral units allow voters to have an equallyweighted vote in the election of representatives, but this ideal is not attainable in practice and many other competing principles are applied simultaneously".
} 


\section{DERECHO ELECTORAL}

De la doctrina citada sobresale la consideración de que si bien no siempre es posible conformar todas las circunscripciones electorales con la misma cantidad de personas electorales para que todas tengan idéntico peso (mucho menos, si se consideraran solamente los votos válidamente emitidos), estas deben configurarse de tal forma que los votos tengan un peso aproximadamente igual, es decir, que sean igualmente considerados en la mayor medida posible. En otras palabras, se busca una igualdad relativa, lo que podría entenderse como que no haya diferencias excesivas.

Hecho el análisis de los instrumentos universales de derechos humanos, queda pendiente una sumaria referencia a otros de ámbito regional. La Declaración Americana de los Derechos y Deberes del Hombre (Bogotá, 1948) establece también un antecedente para el continente americano, escrito de la siguiente forma:

Artículo XX. Toda persona, legalmente capacitada, tiene el derecho de tomar parte en el gobierno de su país, directamente o por medio de sus representantes, y de participar en las elecciones populares, que serán de voto secreto, genuinas, periódicas y libres.

Por su parte, la Convención Americana sobre Derechos Humanos (Organización de Estados Americanos, 1969) sigue una redacción similar a la del artículo 25 del citado Pacto de Nueva York:

Artículo 23. Derechos Políticos

1. Todos los ciudadanos deben gozar de los siguientes derechos y oportunidades:

a) de participar en la dirección de los asuntos públicos, directamente 0 por medio de representantes libremente elegidos;

b) de votar y ser elegidos en elecciones periódicas auténticas, realizadas por sufragio universal e igual y por voto secreto que garantice la libre expresión de la voluntad de los electores, y

c) de tener acceso, en condiciones generales de igualdad, a las funciones públicas de su país. 


\section{DERECHO ELECTORAL}

2. La ley puede reglamentar el ejercicio de los derechos y oportunidades a que se refiere el inciso anterior, exclusivamente por razones de edad, nacionalidad, residencia, idioma, instrucción, capacidad civil o mental, o condena, por juez competente, en proceso penal [énfasis es suplido].

Si bien esta Convención no genera obligaciones para los Estados Unidos de América (caso de estudio del presente artículo), sigue siendo un referente importante para confirmar, en el contexto interamericano, la preponderancia de la igualdad en el ejercicio individual del derecho al sufragio activo.

En cuanto a otras regiones del globo, los instrumentos no suelen ser tan explícitos como los recién citados; sin embargo, pueden derivarse interpretaciones en el mismo sentido de la lectura conjunta de varios de ellos. En el caso de Europa, el artículo 14 del Convenio para la Protección de los Derechos Humanos y de las Libertades Fundamentales (Consejo de Europa, 1950) establece una prohibición de discriminación, que debe dar contenido al derecho a elecciones libres del cuerpo legislativo que se señala en el artículo 3 de su Protocolo Adicional (Consejo de Europa, 1952). Esta circunstancia puede explicarse por la preeminencia de regímenes parlamentarios en el continente, por lo que no necesariamente ha sido esencial tutelar el carácter directo del voto, pues el poder se concentra, principalmente, en los parlamentos, de los cuales y ante los cuales es responsable la jefatura del Gobierno. Al respecto, las directrices adoptadas por la Comisión Europea para la Democracia por el Derecho/ Comisión de Venecia limitan la exigencia de voto directo a: "i. al menos una cámara del Parlamento nacional; ii. los órganos legislativos infranacionales; iii. las asambleas locales" (Comisión Europea para la Democracia por el Derecho, 2011, p. 21).

Para África, la Carta Africana sobre los Derechos Humanos y de los Pueblos (Organización de la Unidad Africana, 1981) establece una fórmula semejante, con la igualdad y la no discriminación de los artículos 2 y 3 como necesarios componentes del derecho a participar en el gobierno, de manera directa o mediante representantes. 


\section{DERECHO EIECTORAL}

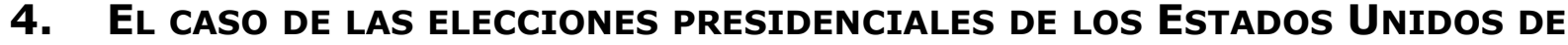 AMÉRICA}

Como se adelantó en la introducción de este trabajo, en los Estados Unidos de América la presidencia y la vicepresidencia son elegidas por voto indirecto en segundo grado, con la intermediación del Colegio Electoral. Este órgano se compone de un número de electores igual a la sumatoria de las representaciones de cada estado en el Congreso -2 por el Senado más el número de congresistas en la Cámara de Representantes, lo cual se fija en proporción de la población según el último censo- más una representación para el Distrito de Columbia, igual a la cantidad más baja de los estados -3 electores en la actualidad-. Esto suma 538 electores, por lo que la elección se gana con 270 votos del Colegio Electoral (la mitad más uno).

Si se utiliza el criterio diferenciador antes propuesto (Rosales, 2014), es claro que la existencia del Colegio Electoral implica que este no solo es un sistema de voto indirecto, sino que no existe igualdad en el voto. Pero a ello se suman algunos problemas de importantes implicaciones políticas.

En casi todas las circunscripciones -consistentes en los estados, junto con el Distrito de Columbia- se adjudica la totalidad de los electores a la tendencia que obtuvo la mayoría de los votos ciudadanos, sean estos tan pocos como 3 escaños, en el caso del Distrito de Columbia, o tantos como 55 , número correspondiente a California ${ }^{5}$. Con esta regla, conocida como "winner takes all", se pierde toda posibilidad de mantener, en la decisión final, una representación proporcional de la diversidad de intenciones de voto dentro de la circunscripción.

Esto se agrava al presentarse el fenómeno conocido como "swing states". Mientras la gran mayoría de los estados mantienen la tendencia de votar por uno u otro partido claramente definido durante varias elecciones, algunos pocos cambian de un proceso al otro, y es difícil predecir cómo votarán. Esto ha ocasionado que la campaña política se haya centrado, por ejemplo, en Pensilvania, Ohio, Florida y Nuevo Hampshire durante las últimas décadas.

\footnotetext{
${ }^{5}$ Son excepciones Maine y Nebraska, en los cuales se adjudican algunos según los resultados de los distritos utilizados para la elección de congresistas.
} 


\section{DERECHO EIECTORAL}

La realidad normativa antes descrita, en la práctica, ha llevado a que de un total de 59 elecciones que se han realizado en esa nación desde 1788-1789, 5 hayan culminado con declarar electas a candidaturas que perdieron la mayoría del voto popular $(1824,1876,1888,2000,2016)$.

Al mantener un sistema de voto indirecto para la elección de la presidencia, se perpetúa una lesión al derecho al sufragio, pues existe una ruptura del principio democrático de una persona, un voto, que se encuentra en la base de la axiología democrática como garantía de igualdad y base de la legitimidad política en la actualidad.

Las últimas encuestas reflejan que la mayoría de la ciudadanía estaría de acuerdo con cambiar el sistema, eliminando el Colegio Electoral, y mutando a elecciones directas. Según las encuestas periódicas de Gallup, en setiembre de 2020 el $61 \%$ de las personas estadounidenses está de acuerdo en abolir el Colegio Electoral (Gallup, 2020). Esto confirma que la falencia no solo se descubre en los instrumentos internacionales y la doctrina, sino que ese mecanismo tampoco cuenta con total aceptación por parte del pueblo que lo está utilizando. Esa crisis de legitimidad del sistema político se manifiesta al observar cómo las elecciones han producido gran polarización política y social en el país (Nohlen, 2007).

Una mirada a fuentes sobre los intentos de dotar de contenido práctico al carácter igualitario del sufragio, en el sentido de acercarse al voto directo, arroja luz sobre las dificultades que ello ha representado. Por ejemplo, ha habido discusiones sobre lo inaceptable que sería para varios países la imposición de un sistema proporcional (Secretaría General de la Organización de las Naciones Unidas et al., 1963). Esto permite entender que es casi imposible que el cambio se geste en el marco del derecho internacional.

En varias ocasiones ha habido iniciativas para reformar ese aspecto del sistema electoral estadounidense. Sin embargo, la mayoría calificada requerida tanto en el Congreso (dos tercios) como en la ratificación de los cuerpos representativos de los estados (tres cuartos) ha vuelto casi imposible su consecución, dada la actual situación política, con gran polarización política y social. En cualquier caso, este problema contemporáneo solamente vislumbra solución en un momento posterior. 


\section{DERECHOELECTORAL}

\section{Hallazgos Y CONCLUSiones}

1) Los instrumentos internacionales sobre derechos humanos relativos al sufragio garantizan la participación electoral de manera universal e igualitaria.

2) La tendencia internacional es la de evolucionar de una concepción de los derechos relativos al sufragio como derecho de los pueblos, a definir derechos individuales para cada persona ciudadana, lo cual ofrece un mayor nivel de protección de la participación política de todas las personas.

3) Dada la naturaleza del Pacto Internacional de Derechos Civiles y Políticos, así como la redacción de sus artículos, se entiende que estos derechos políticos son dirigidos a las personas ciudadanas, no así a los estados o las circunscripciones electorales. Esto establece obligaciones directamente exigibles a los estados por parte de las personas ciudadanas.

4) Del Pacto Internacional de Derechos Civiles y Políticos y del consenso internacional, se entiende como rasgo esencial de la democracia en los procesos de elección que el voto se realice de manera directa, y no con órganos que funcionen como intermediarios y diluyan la voluntad de la mayoría popular. Lo anterior se concreta en el principio democrático de "una persona, un voto".

5) El voto indirecto, en la práctica, implica una inequidad en cuanto al peso del voto de cada persona ciudadana, pues la designación de electores no será proporcional.

6) Si bien el carácter directo del voto puede entenderse como derecho humano, en el caso de los regímenes parlamentarios ese requisito puede entenderse satisfecho siempre que la jefatura del Gobierno integre el parlamento por haber sido elegido de manera directa, y sea responsable ante ese mismo órgano.

7) En el caso de las elecciones presidenciales de los Estados Unidos de América, la presidencia y la vicepresidencia de la nación son elegidas en segundo grado por un Colegio Electoral que se constituye, en el primer grado, en elecciones en las que se presentan graves diferencias en el peso del voto de cada persona, según la circunscripción electoral en la que se encuentre. Esto ha impedido que el voto popular de la 


\section{DERECHO EIECTORAL}

mayoría sea el que determine el resultado de las elecciones en muchas ocasiones. Este sistema responde a las necesidades políticas de otra época, en la cual no se tenía claridad sobre los derechos de participación política como derecho humano. Esta circunstancia requiere una evolución hacia voto directo, para ajustar el sistema electoral de uno de los funcionarios públicos más poderosos del mundo, en una de las democracias más antiguas y consolidadas, a los paradigmas contemporáneos de derechos humanos.

\section{REFERENCIAS BIBLIOGRÁFICAS}

Aragón, M. (2007). Derecho de sufragio: Principio y función. En D. Nohlen, D. Zovatto, J. Orozco y J. Thompson (Eds.), Tratado de derecho electoral comparado de América Latina (2. ${ }^{a}$ ed.) (pp. 162-177). San José: Instituto Interamericano de Derechos Humanos.

Brenes, L. D., y Matarrita, M. A. (2012). Efectos colaterales del voto preferente. Revista de Derecho Electoral, (14), 42-62.

Comisión Europea para la Democracia por el Derecho. (2011). Código de buenas prácticas en materia electoral: Directrices e informe explicativo. México: Tribunal Electoral del Poder Judicial de la Federación. Recuperado de https://www.te.gob.mx/sites/default/files/informacion_importante/2012/04 /codigo_buenas_practicas_pdf_18140.pdf

Comité de Derechos Humanos de la Organización de las Naciones Unidas. (1996). Comentario General n. ${ }^{\circ} 25$. CCPR/C/21/Rev.1/Add.7. 27 de agosto de 1996 . Recuperado de https://documents-ddsny.un.org/doc/UNDOC/GEN/G96/180/97/PDF/G9618097.pdf?OpenElement

Consejo de Europa. (1950). Convenio para la Protección de los Derechos Humanos y de las Libertades Fundamentales. Recuperado de https://www.derechoshumanos.net/Convenio-Europeo-de-DerechosHumanos-CEDH/

Consejo de Europa (1952). Protocolo Adicional para la Protección de los Derechos Humanos y de las Libertades Fundamentales. Recuperado de https://www.acnur.org/fileadmin/Documentos/BDL/2002/1253.pdf?view=1

Fox, G. H. (1992). The Right to Political Participation In International Law. Yale Journal of International Law, (17), 539-607. 


\section{DERECHO ELECTORAL}

Gallup. (2020, septiembre 24). 61 \% of Americans Support Abolishing Electoral College. Recuperado de https://news.gallup.com/poll/320744/americanssupport-abolishing-electoral-college.aspx

Instituto Interamericano de Derecho Humanos. (2017). Diccionario electoral: Vol. $I-I I$ (3. ${ }^{a}$ ed.). Instituto Interamericano de Derechos Humanos.

Instituto Internacional para la Democracia y la Asistencia Electoral. (2002). International Electoral Standards: Guidelines for reviewing the legal framework of elections. Suecia: Instituto Internacional para la Democracia y la Asistencia Electoral.

National Archives. (agosto 262020,). Electoral College Results. National Archives. Recuperado de https://www.archives.gov/electoral-college/results

Nohlen, D. (2007). Sistemas electorales presidenciales y parlamentarios. En D. Nohlen, D. Zovatto, J. Orozco y J. Thompson (Eds.), Tratado de derecho electoral comparado de América Latina (2. ${ }^{a}$ ed., pp. 294-333). San José: Instituto Interamericano de Derechos Humanos.

Organización de la Unidad Africana (1981). Carta Africana sobre los Derechos Humanos y de los Pueblos (Carta de Banjul). Recuperado de https://www.acnur.org/fileadmin/Documentos/BDL/2002/1297.pdf

Organización de las Naciones Unidas. (1948). Declaración Universal de Derechos Humanos. Recuperado de https://www.un.org/es/about-us/universaldeclaration-of-human-rights

Organización de las Naciones Unidas. (1966). Pacto Internacional de los Derechos Civiles y Políticos. Recuperado de https://www.ohchr.org/sp/professionalinterest/pages/ccpr.aspx

Organización de los Estados Americanos. (1969). Convención Americana sobre Derechos Humanos. Recuperado de https://www.oas.org/dil/esp/tratados b32 convencion americana sobre derechos humanos.htm

Picado, S. (2007). Derechos políticos como derechos humanos. En D. Nohlen, D. Zovatto, J. Orozco y J. Thompson (Eds.), Tratado de derecho electoral comparado de América Latina (2. ${ }^{a}$ ed., pp. 48-59). San José: Instituto Interamericano de Derechos Humanos.

Rosales, C. M. (2014). Radiografía de la democracia: Naturaleza, fundamentos y objetivos. Revista de Derecho Electoral, (18), 86-130. 


\section{DERECHO ELECTORAL}

Secretaría General de la Organización de las Naciones (1963). Draft Principles on Freedom and Non-Discrimination in the Matter of Political Rights: Note. Recuperado de https://digitallibrary.un.org/record/643414?ln=es\# recordfiles-collapse-header

United Nations Centre for Human Rights. (1994). Human Rights and Elections: a handbook on the Legal, Technical and Human Rights Aspects of Elections. New York: United Nations. 